\title{
Development of a FHIR Layer on Top of the OMOP Common Data Model for the CAPABLE Project
}

\author{
Matteo GABETTA ${ }^{\mathrm{a}, 1}$, Anna ALLONI ${ }^{\mathrm{a}}$, Francesca POLCE ${ }^{\mathrm{b}}$, Giordano LANZOLA $^{\mathrm{b}}$, \\ Enea PARIMBELLI ${ }^{\mathrm{b}}$ and Nicola BARBARINI ${ }^{\mathrm{a}}$ \\ ${ }^{a}$ BIOMERIS (BIOMEdical Research Informatics Solutions), Pavia, Italy \\ ${ }^{\mathrm{b}}$ Department of Electrical, Computer and Biomedical Engineering, University of \\ Pavia, Pavia, Italy
}

Keywords. OMOP, HL7 FHIR, API, Cancer

\section{Introduction}

The CAPABLE (CAncer PAtient Better Life Experience) project [1], funded in the $\mathrm{H} 2020$ program $^{2}$, is developing a novel system to improve the quality of life of cancer patients managed at home. CAPABLE system is based on a distributed software architecture where different components cooperate with the aim of "early detecting and managing cancer-related issues and at satisfying the needs of patients and their home caregivers". One of the core CAPABLE components is the "CAPABLE Data Platform" (DP); the main objective of the DP is to provide a persistent layer where to store and fetch all project's patient-related data.

To guarantee a state-of-the art level component, OMOP [2] Common Data Model (CDM) and HL7-FHIR [3] have been chosen for persistency and exchange format respectively. The main reason for choosing OMOP is due to its "Standardized clinical data" tables, which are designed to hold disparate patient-related data, and to the "Standardized vocabularies", a set of international standard terminologies which are consolidated into the same code system. Alongside with the need of having a standard model to represent persisted data, the project also needed a reliable format for exchanging those data in a web-service safe mode: FHIR was chosen for this purpose because it is based on a "composition approach", representing standard clinical entities as resources that can be combined with each other.

The omoponfhir open-source project [4] constituted the starting point for the development of the DP (which in fact can be considered a fork of this project); in this article we highlight the main changes/additions and customization of omoponfhir to make it fit the aims and requirements of the CAPABLE project.

1 Corresponding Author, BIOMERIS, Via Ferrata 1, 27100 Pavia, Italy; E-mail: matteo.gabetta@biomeris.it.

2 The work described in this article has been funded by the European Union's Horizon 2020 research and innovation programme under grant agreement No 875052 - CAPABLE 


\section{Methods}

The development of DP is covering several aspects of the omoponfhir project; the most relevant general improvements concern: (i) an easier management of FHIR Coding resource, that is used by mostly all other resources; (ii) the option of automatically translating any FHIR Coding to its standard OMOP synonym (if present), thus allowing the interoperability between different terminologies directly in FHIR; (iii) the possibility to go beyond a the flat searching model, where all constraints are evaluated in sequence, with the introduction of a hierarchical query model.

For what concerns the supported FHIR Resources, several new search parameters have been implemented in the code base; moreover, new resources, that are not managed by the original project, have been implemented: Goal, List and Communication.

Another relevant improvement is related to the FHIR Observation Resource, which can represent concepts that map multiple OMOP domains (e.g., Observation, Measurement, Procedure and Condition Occurrence): a fine-grained management has been implemented, taking also into account the fact that an Observation could be possibly negated or further specified by scales or grades.

The OMOP CDM has been also extended with new tables; the most relevant one is f_update and allows to specify the lastupdated attribute for all the facts that can be stored in the DP: this is a crucial information for the CAPABLE system and for FHIR in general that wasn't manageable with the standard OMOP CDM. Finally, the CTCAE (Common Terminology Criteria for Adverse Events) [5] is in the process of being added to the OMOP Vocabularies and mapped to standard OMOP concepts.

\section{Preliminary Results and Next Steps}

The overall CAPABLE system, DP included, has already undergone two development iterations each one concluded with a live demonstration. The demonstrations, designed together with clinicians and patients involved in the project, revolve around prototypical fictional patients, following them in different settings (enrollment visit, follow-up, homecare) and focus on specific clinical guidelines.

During the demonstrations, mostly all of the CAPABLE components have interacted with DP by storing and fetching more than 130 different FHIR Resources, which have been saved and then retrieved from the customized OMOP CDM.

Next steps involve the release in production of the DP to comply with the all the project's use cases.

\section{References}

[1] CAPABLE H2020 Project [Internet]. Capable. 2021 [cited 2021Jul26]. Available from: https://capableproject.eu/

[2] The OHDSI community. (2020). The Book of OHDSI (Version 2020-10-19). The Book of OHDSI (p. 470). Zenodo. http://doi.org/10.5281/zenodo.4265256

[3] FHIR v4.0.1 [Internet]. H17.org. 2021 [cited 26 July 2021]. Available from: https://www.hl7.org/fhir/

[4] Choi, M., Starr, R., Braunstein, M., \& Duke, J. (2016). OHDSI on FHIR platform development with OMOP CDM mapping to FHIR Resources. In OHDSI Symposium, Observational Health Data Sciences and Informatics, Washington, DC.

[5] CTCAE [Internet]. NCI. $2021 \quad$ [cited 26 July 2021]. Available from https://ctep.cancer.gov/protocoldevelopment/electronic_applications/ctc.htm 